\title{
Imaging cerebral microemboli during TAVI
}

Cerebral microembolization is

inherent to transcatheter aortic valve implantation (TAVI), and occurs with balloon-expandable and self-expandable prostheses, with transfemoral (TF) or transapical (TA) access, and during all stages of the procedure. These findings from a single-center study have been reported in Circulation by researchers from Germany. However, in this investigation, microembolization did not lead to long-term neurological deficits or cognitive impairment.

TAVI is known to be associated with an increased risk of stroke and clinically silent ischemia compared with surgical aortic valve replacement. Manipulation of the aorta and calcified valve during TAVI, which releases atherosclerotic debris into the circulation, is thought to account for this increased neurological risk. Kahlert et al. aimed to determine the origin of microembolization, and subsequent outcomes, in a series of 83 elderly (mean age 80.6 years) patients undergoing TAVI for severe, symptomatic aortic stenosis.

Between August 2009 and May 2011, 58 patients underwent TF TAVI; 32 with the self-expandable Corevalve (Medtronic, Luxembourg) and 26 with the balloon-expandable SAPIEN XT ${ }^{\oplus}$ valve (Edwards Lifesciences Corporation, Irvine, CA, USA). In addition, 25 patients had TA TAVI with the Edwards valve. Transcranial Doppler examination was performed at baseline, during TAVI, and at 3 months after the procedure to identify high-intensity transient signals (HITS) - a marker of microembolization. All patients had mild (87\%) or moderate (13\%) aortic atheromata; carotid artery stenosis of $30-70 \%$ and $>70 \%$ luminal narrowing was noted in $33 \%$ and $2 \%$ of patients, respectively; and $24 \%$ of patients had permanent atrial fibrillation.

HITS occurred in all patients during all stages of TAVI. The highest proportion of HITS occurred during positioning and implantation of the prosthesis, when the calcified native valve was being manipulated. Overall, no significant differences in the number of HITS was observed between the three treatment groups. However, a trend was noted towards an increased number of HITS in patients whose procedures took longer, supporting the "time is brain" concept.

Periprocedural stroke occurred in two patients who had TF TAVI with the Corevalve ${ }^{\varpi}$. One patient died and the other recovered fully with no neurological sequelae at 3 months. None of the patients experienced a decline in neurocognitive function during the follow-up period. "Our findings call for strategies to reduce the risk of procedural embolization during TAVI," says Dr Kahlert. "It will be particularly interesting to monitor the effects of new embolic protection devices on the frequency of clinically apparent neurological events, of intraprocedural embolic signals on transcranial Doppler, and of new lesions on postprocedural MRI."

\section{Alexandra King}

Original article Kahlert, P. et al. Cerebral embolization during transcatheter aortic valve implantation: a transcranial Doppler study. Circulation doi:10.1161/ CIRCULATIONAHA.112.092544 